\title{
ON A 2-DIMENSIONAL EINSTEIN KAEHLER SUBMANIFOLD OF A COMPLEX SPACE FORM
}

\author{
YOSHIO MATSUYAMA
}

\begin{abstract}
In this paper we consider when a Kaehler submanifold of a complex space form is Einstein with respect to the induced metric. Then we shall show that (1) a 2-dimensional complete Kaehler submanifold $M$ of a 4-dimensional complex projective space $P^{4}(C)$ is Einstein if and only if $M$ is holomorphically isometric to $P^{2}(C)$ which is totally geodesic in $P^{4}(C)$ or a hyperquadric $Q^{2}(C)$ in $P^{3}(C)$ which is totally geodesic in $P^{4}(C)$, and that (2) if $M$ is a 2-dimensional Einstein Kaehler submanifold of a 4-dimensional complex space form $\tilde{M}^{4}(\tilde{c})$ of nonpositive constant holomorphic sectional curvature $\tilde{c}$, then $M$ is totally geodesic.
\end{abstract}

Let $M^{n}$ be an $n$-dimensional Kaehler submanifold of an $(n+p)$-dimensional complex space form $\tilde{M}^{n+p}(\tilde{c})$ of constant holomorphic sectional curvature $\tilde{c}$. Let $P^{n+p}(C)$ be an $(n+p)$-dimensional complex projective space with the Fubini-Study metric of constant holomorphic sectional curvature 1, and let $C^{n+p}$ (or $D^{n+p}$ ) be a complex $(n+p)$-dimensional Euclidean space with the usual Hermitian metric (resp. the $(n+p)$-dimensional open unit ball in $C^{n+p}$ with the natural complex structure and the Bergman metric of constant holomorphic sectional curvature -1). Any $m$-dimensional complex space form is (after multiplying the metric by a suitable constant) locally complex analytically isometric to $P^{m}(C), C^{m}$ or $D^{m}$, according as the holomorphic sectional curvature is positive, zero or negative. We consider the following question:

When is $M$ Einstein with respect to the induced metric?

The answer is known in the following cases:

(1) $p=1$ (Smyth [8]).

A complete hypersurface $M$ of $P^{n+1}(C)$ is Einstein if and only if $M$ is holomorphically isometric to $P^{n}(C)$ or a complex hyperquadric $Q^{n}(C)$.

A complete and simply connected hypersurface $M$ of $C^{n+1}$ (resp. $D^{n+1}$ ) is Einstein if and only if $M$ is holomorphically isometric to $C^{n}$ (resp. $D^{n}$ ).

(2) A complete intersection submanifold (Hano [3]).

A complete intersection submanifold $M^{n}$ of $P^{n+p}(C)$ is Einstein if and only if $M$ is $P^{n}(C)$ which is totally geodesic in $P^{n+p}(C)$ or a hyperquadric $Q^{n}(C)$ in $P^{n+1}(C)$ which is totally geodesic in $P^{n+p}(C)$.

Received by the editors April 2, 1983 and, in revised form, February 22, 1984.

1980 Mathematics Subject Classification. Primary 53B25, 53C40.

Kev words and phrases. Einstein Kaehler submanifolds, complex space forms, second fundamental forms. 
In this paper we consider the case of $n=2$. We show

TheOREM A. A complete Kaehler submanifold $M^{2}$ of $P^{4}(C)$ is Einstein if and only if $M$ is holomorphically isometric to $P^{2}(C)$ which is totally geodesic in $P^{4}(C)$ or a hyperquadric $Q^{2}(C)$ in $P^{3}(C)$ which is totally geodesic in $P^{4}(C)$.

Since for each $n \geqslant 1$ there exists a Kaehler imbedding $f: P_{1 / 2}^{n}(C) \rightarrow$ $P^{n+(n(n+1)) / 2}(C)$ (O'Neill [7]), the codimension of Theorem A is best possible, where $1 / 2$ of $P_{1 / 2}^{n}(C)$ denotes constant holomorphic sectional curvature.

THEOREM B. Let $M^{2}$ be an Einstein Kaehler submanifold immersed in $\tilde{M}^{4}(\tilde{c}), \tilde{c} \leqslant 0$. Then $M$ is totally geodesic.

1. Kaehler submanifolds. As in the introduction, let $M^{n}$ be a Kaehler submanifold of $\tilde{M}^{n+p}(\tilde{c})$. And let $f$ be its Kaehler immersion (i.e., holomorphically isometric immersion). Let $J_{0}$ (resp. $J$ ) be the complex structure of $M$ (resp. $\tilde{M}$ ) and let $g_{0}$ (resp. $g$ ) be the Kaehler metric of $M$ (resp. $\tilde{M}$ ). In order to simplify the presentation we identify, for each $x \in M$, the tangent space $T_{x}(M)$ with $f_{*}\left(T_{x}(M)\right) \subset T_{f(x)}(\tilde{M})$ by means of $f_{*}$. The normal space $T_{x}^{\perp}(M)$ is the subspace of $T_{f(x)}(\tilde{M})$ consisting of all $X \in T_{f(x)}(\tilde{M})$ which are orthogonal, with respect to $g$, to the subspace $f_{*}\left(T_{x}(M)\right)$. Since $f^{*} g=g_{0}$ and $J \circ f_{*}=f_{*} \circ J_{0}$, where $J_{0}$ is the almost complex structure of $M$, the structures $g_{0}$ and $J_{0}$ on $T_{x}(M)$ are respectively identified with the restrictions of the structures $g$ and $J$ to the subspace $f_{*}\left(T_{x}(M)\right)$. With this identification in mind we drop the subscript 0 on $g_{0}$ and $J_{0}$. Let $\nabla$ (resp. $\tilde{\nabla}$ ) denote the covariant differentiation in $M$ (resp. $\tilde{M}$ ), and let $\nabla^{\perp}$ denote covariant differentiation in the normal bundle.

With each $\xi \in T_{x}^{\perp}(M)$ is associated a linear transformation of $T_{x}(M)$ in the following way. Extend $\xi$ to a normal vector field defined in a neighborhood of $x$ and define $-A_{\xi} X$ to be the tangential component of $\tilde{\nabla}_{X} \xi$ for $X \in T_{x}(M) . A_{\xi} X$ depends only on $\xi$ at $x$ and $X$. Given an orthonormal basis $\xi_{1}, \ldots, \xi_{p}, \xi_{1^{*}}=J \xi_{1}, \ldots, \xi_{p^{*}}=J \xi_{p}$ of $T_{x}^{\perp}(M)$. We write $A_{\lambda}=A_{\xi_{\lambda}}$ and call the $A_{\lambda}$ 's the second fundamental forms associated with $\xi_{1}, \ldots, \xi_{p}, \xi_{1^{*}}, \ldots, \xi_{p^{*}}$ are now orthonormal normal vector field in a neighborhood $U$ of $x$, they determine normal connection forms $s_{\alpha \beta}, t_{\alpha \beta}$ in $U$ by

$$
\nabla_{X}^{\perp} \xi_{\alpha}=\sum_{\beta} s_{\beta \alpha}(X) \xi_{\beta}+\sum_{\beta} t_{\beta \alpha}(X) \xi_{\beta^{*}}, \quad s_{\alpha \beta}+s_{\beta \alpha}=0, t_{\alpha \beta}-t_{\beta \alpha}=0
$$

for $X \in T_{x}(M)$. Let $S$ and $\rho$ be the Ricci tensor and the scalar curvature for $M$, respectively. Then we have the following relationship (in this paper $\alpha, \beta, \gamma$ run from 1 to $p$ and $\lambda, \mu$ run from $1, \ldots, p, 1^{*}, \ldots, p^{*}$, except when noted) [5]:

$$
\begin{gathered}
\tilde{\nabla}_{X} Y=\nabla_{X} Y+\sigma(X, Y), \\
\sigma(X, Y)=\sum g\left(A_{\lambda} X, Y\right) \xi_{\lambda}, \\
g\left(A_{\alpha} X, Y\right)=g\left(A_{\alpha} Y, X\right), \quad A_{\alpha^{*}}=J A_{\alpha}, \quad A_{\alpha} J=-A_{\alpha} J \\
\left(\nabla_{X} A_{\alpha}\right) Y-\sum_{\beta} s_{\beta \alpha}(X) A_{\beta} Y-\sum_{\beta} t_{\beta \alpha}(X) J A_{\beta} Y \\
=\left(\nabla_{Y} A_{\alpha}\right) X-\sum_{\beta} s_{\beta \alpha}(Y) A_{\beta} X-\sum_{\beta} t_{\beta \alpha}(Y) J A_{\beta} X \text {-Codazzi equation, }
\end{gathered}
$$




$$
\begin{gathered}
S=\frac{n+1}{2} \tilde{c} I-2 \sum_{\alpha} A_{\alpha}^{2}, \\
\rho=n(n+1) \tilde{c}-\|\sigma\|^{2},
\end{gathered}
$$

where $X, Y$ are tangent to $M, \sigma$ is also called the second fundamental form of $f$, and $I,\|\sigma\|$ denote the identity transformation of $T_{x}(M)$, the length of $\sigma$, so that

$$
\|\sigma\|^{2}=2 \sum \operatorname{trace} A_{\alpha}^{2} .
$$

2. Proposition. Let $M^{n}$ be a Kaehler submanifold of $\tilde{M}^{n+p}(\tilde{c})$.

Definition. For $x \in M$, the first normal space, $N_{1}(x)$, is the orthogonal complement in $T_{x}(M)$ of the set $N_{0}(x)=\left\{\xi \in T_{x}(M) \mid A_{\xi}=0\right\}$.

Proposition 1. Let $M^{2}$ be a 2-dimensional Einstein Kaehler submanifold of $\tilde{M}^{2+2}(\tilde{c})$. Then $\operatorname{dim} N_{1}(x) \neq 2$.

Proof. We assume that $\operatorname{dim} N_{1}(x)=2$. Then $\operatorname{dim} N_{1}(y) \geqslant 2$ in a neighborhood $U_{0}$ of $x$. In terms of codimension $M=2$ we have $\operatorname{dim} N_{1}(y)=2$ in $U_{0}$. Choose local orthonormal vector fields $\xi_{1}, \xi_{2}, J \xi_{1}, J \xi_{2}$ so that a symmetric $(4,4)$-matrix (trace $\left.A_{\lambda} A_{\mu}\right)$ can be diagonalized.

Let $\Omega=\left\{e_{1}, e_{2}, e_{1^{*}}=J e_{1}, e_{2^{*}}=J e_{2}\right\}$ be an orthonormal basis for $T_{x}(M)$ with respect to which $A_{1}$ is diagonal of the form

By means of

$$
A_{1}=\left(\begin{array}{cccc}
\alpha & & & \\
& \beta & & \\
& & -\alpha & -\beta
\end{array}\right) .
$$

$$
\begin{aligned}
& g\left(A_{2} e_{1}, e_{2^{*}}\right)=g\left(A_{2} e_{2}, e_{1^{*}}\right), \\
& g\left(A_{2} e_{a^{*}}, e_{b^{*}}\right)=-g\left(A_{2} e_{a}, e_{b}\right), \quad a, b=1,2,
\end{aligned}
$$

$A_{2}$ is represented by the matrix

$$
A_{2}=\left(\begin{array}{llll}
a_{11} & a_{12} & a_{11^{*}} & a_{12^{*}} \\
a_{12} & a_{22} & a_{12^{*}} & a_{22^{*}} \\
a_{11^{*}} & a_{12^{*}} & -a_{11} & -a_{12} \\
a_{12^{*}} & a_{22^{*}} & -a_{12} & -a_{22}
\end{array}\right) .
$$

Since $M^{2}$ is Einstein, i.e., $S=(\rho /(2 \operatorname{dim} M)) I$, we have

$$
A_{1}^{2}+A_{2}^{2}=\frac{1}{8}\|\sigma\|^{2} I .
$$

From trace $A_{1} A_{2}=\operatorname{trace} A_{1} A_{2^{*}}=0$ we have

$$
\begin{gathered}
a_{11^{\alpha}} \alpha+a_{22} \beta=0, \\
a_{11^{*}} \alpha+a_{22^{*}} \beta=0 .
\end{gathered}
$$

Case 1. $a_{11}=a_{22}=a_{11^{*}}=a_{22^{*}}=0$. Then $A_{2}^{2}=\left(a_{12}^{2}+a_{12^{*}}^{2}\right) I$. Thus from (2.1) we obtain $\alpha^{2}=\beta^{2}$, i.e., $A_{1}^{2}=\alpha^{2} I$. Then

$$
A_{1} \circ A_{\xi_{1}+\xi_{2}}^{2}=A_{\xi_{1}+\xi_{2}}^{2} \circ A_{1}
$$




$$
\begin{aligned}
& A_{1} \circ A_{\xi_{1}+J \xi_{2}}^{2}=A_{\xi_{1}+J \xi_{2}}^{2} \circ A_{1}, \\
& A_{\xi_{1}+\xi_{2}}^{2} \circ A_{\xi_{1}+J \xi_{2}}^{2}=A_{\xi_{1}+J \xi_{2}}^{2} \circ A_{\xi_{1}+\xi_{2}}^{2} \circ
\end{aligned}
$$

Hence $A_{1}, A_{\xi_{1}+\xi_{2}}^{2}$ and $A_{\xi_{1}+J \xi_{2}}^{2}$ can be simultaneously diagonalized. We may assume that from the beginning we choose such a basis $\Omega$. Then we obtain

$$
\begin{aligned}
& A_{\xi_{1}+\xi_{2}}^{2}-A_{1}^{2}-A_{2}^{2}=A_{1} A_{2}+A_{2} A_{1} \\
& =\left(\begin{array}{cccc}
0 & a_{12} \alpha+a_{12} \beta & 0 & a_{12^{*}} \alpha-a_{12^{*}} \beta \\
a_{12} \alpha+a_{12} \beta & 0 & -a_{12^{*}} \alpha+a_{12^{*}} \beta & 0 \\
0 & -a_{12^{*}} \alpha+a_{12^{*}} \beta & 0 & a_{12} \alpha+a_{12} \beta \\
a_{12^{*}} \alpha-a_{12^{*}} \beta & 0 & a_{12} \alpha+a_{12} \beta & 0
\end{array}\right), \\
& A_{\xi_{1}+J \xi_{2}}^{2}-A_{1}^{2}-A_{2}^{2} \\
& =\left(\begin{array}{cccc}
0 & & 0 & a_{12} \alpha-a_{12} \beta \\
-a_{12^{*}} \alpha-a_{12^{*}} \beta & 0 & -a_{12^{*}} \alpha-a_{12^{*}} \beta & 0 \\
0 & -a_{12} \alpha+a_{12} \beta & 0 & 0 \\
a_{12} \alpha-a_{12} \beta & 0 & -a_{12^{*} \alpha-a_{12 *} \beta} \beta & 0
\end{array}\right) .
\end{aligned}
$$

Therefore

$$
\begin{aligned}
& a_{12} \alpha+a_{12} \beta=0, \quad a_{12} \alpha-a_{12} \beta=0, \\
& a_{12^{*}} \alpha+a_{12^{*}} \beta=0, \quad a_{12^{*}} \alpha-a_{12^{*}} \beta=0 .
\end{aligned}
$$

Since we may assume that $(\alpha \beta) \neq 0$, we obtain $a_{12}=a_{12^{*}}=0$. This contradicts the assumption that $k=2$.

Case 2. $\left(a_{11} a_{22} a_{11^{*}} a_{22^{*}}\right) \neq 0$. Since $A_{2}^{2}$ is diagonal, we obtain

$$
\begin{aligned}
& \left(a_{11}+a_{22}\right) a_{12}+\left(a_{11^{*}}+a_{22^{*}}\right) a_{12^{*}}=0, \\
& \left(a_{22^{*}}-a_{11^{*}}\right) a_{12}+\left(a_{11}-a_{22}\right) a_{12^{*}}=0 .
\end{aligned}
$$

If $\left(a_{12} a_{12^{*}}\right) \neq 0$, i.e., $a_{11}^{2}+a_{11^{*}}^{2}=a_{22}^{2}+a_{22^{*}}^{2}$, then we can put $A_{2}^{2}=a^{2} I$, where

$$
a^{2}=a_{11}^{2}+a_{12}^{2}+a_{11^{*}}^{2}+a_{12^{*}}^{2}=a_{22}^{2}+a_{12}^{2}+a_{22^{*}}^{2}+a_{12^{*}}^{2} .
$$

Combining this fact with (2.1), we have $\alpha^{2}=\beta^{2}$, i.e., $A_{1}^{2}=\alpha^{2} I$. Then we have

$$
\begin{aligned}
& A_{1} \circ A_{\xi_{1}+\xi_{2}}^{2}=A_{\xi_{1}+\xi_{2}}^{2} \circ A_{1}, \\
& A_{1} \circ A_{\xi_{1}+J \xi_{2}}^{2}=A_{\xi_{1}+J \xi_{2}}^{2} \circ A_{1}, \\
& A_{\xi_{1}+\xi_{2}}^{2} \circ A_{\xi_{1}+J \xi_{2}}^{2}=A_{\xi_{1}+J \xi_{2}}^{2} \circ A_{\xi_{1}+\xi_{2}}^{2} .
\end{aligned}
$$

Thus by the same argument as Case 1 we know that the case of $\left(a_{12} a_{12^{*}}\right) \neq 0$ cannot occur. Thus we may assume that $a_{12}=a_{12^{*}}=0$. Then from (2.1) we have

$$
\begin{aligned}
& \alpha^{2}+a_{11}^{2}+a_{11^{*}}^{2}=\frac{1}{8}\|\sigma\|^{2}, \\
& \beta^{2}+a_{22}^{2}+a_{22^{*}}^{2}=\frac{1}{8}\|\sigma\|^{2} .
\end{aligned}
$$

Multiplying (2.4) (resp. (2.5)), by $\alpha^{2}$ (resp. $\beta^{2}$ ) we have

$$
\begin{aligned}
& \alpha^{4}+a_{11}^{2} \alpha^{2}+a_{11^{*}}^{2} \alpha^{2}=\frac{1}{8} \alpha^{2}\|\sigma\|^{2}, \\
& \beta^{4}+a_{22}^{2} \beta^{2}+a_{22^{*}}^{2} \beta^{2}=\frac{1}{8} \beta^{2}\|\sigma\|^{2} .
\end{aligned}
$$


Using (2.2) and (2.3), we get from (2.6) that

$$
\alpha^{4}+a_{22}^{2} \beta^{2}+a_{22 *}^{2} \beta^{2}=\frac{1}{8} \alpha^{2}\|\sigma\|^{2} .
$$

Subtracting (2.7) from $(2.6)^{\prime}$, we obtain

$$
\left(\alpha^{2}-\beta^{2}\right)\left(\alpha^{2}+\beta^{2}-\frac{1}{8}\|\sigma\|^{2}\right)=0 .
$$

If $\alpha^{2} \neq \beta^{2}$, then from (2.4) and (2.5) we have

$$
A_{2}^{2}=\left(\begin{array}{llll}
\beta^{2} & & & \\
& \alpha^{2} & & \\
& & \beta^{2} & \\
& & & \alpha^{2}
\end{array}\right) .
$$

Hence we know that $\operatorname{det} A_{1}^{2}=\operatorname{det} A_{2}^{2}$. Since we have

$$
\operatorname{det} A_{1}=\alpha^{2} \beta^{2}, \quad \operatorname{det} A_{2}=a_{11}^{2} a_{22}^{2}+a_{11^{2}}^{2} a_{22^{*}}^{2}+a_{11^{*}}^{2} a_{22}^{2}+a_{11^{*}}^{2} a_{22^{*}}^{2},
$$

we obtain

$$
\alpha^{2} \beta^{2}=a_{11}^{2} a_{22}^{2}+a_{11}^{2} a_{22^{*}}^{2}+a_{11^{*}}^{2} a_{22}^{2}+a_{11^{*}}^{2} a_{22^{*}}^{2} .
$$

On the other hand, from (2.2) and (2.3) we have

$$
a_{11} a_{22^{*}}-a_{11^{*}} a_{22}=0 \text {, }
$$

since we may assume that $(\alpha \beta) \neq 0$. Combining (2.8) and (2.9), we obtain

$$
\alpha^{2} \beta^{2}=\left(a_{11} a_{22}+a_{11^{*}} a_{22^{*}}\right)^{2} .
$$

If $\alpha \beta=a_{11} a_{22}+a_{11^{*}} a_{22^{*}}$, then multiplying (2.2) by $\alpha$ and using (2.9), we have

$$
a_{11}\left(a_{22}^{2}+a_{22^{*}}^{2}\right)=0 ; \text { i.e., } \quad a_{11} \alpha^{2}=0 \text {. }
$$

Similarly, we have

$$
\begin{array}{lll}
a_{22}\left(a_{11}^{2}+a_{11^{*}}^{2}\right)=0 ; & \text { i.e., } & a_{22} \beta^{2}=0, \\
a_{11^{*}}\left(a_{22}^{2}+a_{22^{*}}^{2}\right)=0 ; & \text { i.e., } & a_{11^{*}} \alpha^{2}=0, \\
a_{22^{*}}\left(a_{11}^{2}+a_{11^{*}}^{2}\right)=0 ; & \text { i.e., } & a_{22^{*}} \beta^{2}=0 .
\end{array}
$$

Hence, we obtain

$$
\left(\begin{array}{cccc}
\alpha^{2} & & & \\
& \beta^{2} & & \\
& & \alpha^{2} & \\
& & & \beta^{2}
\end{array}\right)\left(\begin{array}{c}
a_{11} \\
a_{22} \\
a_{11^{*}} \\
a_{22^{*}}
\end{array}\right)=0 .
$$

Since $\left(a_{11} a_{22} a_{11^{*}} a_{22^{*}}\right) \neq 0$, we have $\alpha \beta=0$. Assume that $\alpha \beta \neq 0$. Then from (2.10) we have $\alpha \beta+a_{11} a_{22}+a_{11^{*}} a_{22^{*}}=0$ and

$$
\begin{aligned}
& \sigma\left(e_{1}, e_{1}\right)=\alpha \xi_{1}+a_{11} \xi_{2}-a_{11^{*}} \xi_{2^{*}}, \\
& \sigma\left(e_{2}, e_{2}\right)=\beta \xi_{1}+a_{22} \xi_{2}-a_{22^{*}} \xi_{2^{*}} .
\end{aligned}
$$

Hence, we obtain

$$
g\left(\sigma\left(e_{1}, e_{1}\right), \sigma\left(e_{2}, e_{2}\right)\right)=g\left(\sigma\left(e_{1}, e_{1}\right), \sigma\left(e_{2}, e_{2^{*}}\right)\right)=0
$$

and $\sigma\left(e_{1}, e_{2}\right)=0$. On the other hand, from (2.1) we have

$$
\left\|\boldsymbol{\sigma}\left(E_{a}, E_{a}\right)\right\|^{2}+\left\|\boldsymbol{\sigma}\left(E_{1}, E_{2}\right)\right\|^{2}=\frac{1}{8}\|\boldsymbol{\sigma}\|^{2}, \quad a=1,2,
$$




$$
\begin{gathered}
g\left(\sigma\left(E_{1}, E_{1}\right), \sigma\left(E_{1}, E_{2}\right)\right)+g\left(\sigma\left(E_{2}, E_{2}\right), \sigma\left(E_{1}, E_{2}\right)\right)=0, \\
g\left(\sigma\left(E_{1}, E_{1}\right), \sigma\left(E_{1}, E_{2^{*}}\right)\right)-g\left(\sigma\left(E_{2}, E_{2}\right), \sigma\left(E_{1}, E_{2^{*}}\right)\right)=0,
\end{gathered}
$$

where $\left\{E_{1}, E_{2}, E_{1^{*}}, E_{2^{*}}\right\}$ is a suitable local orthonormal frame around $x$ such that $E_{j}=e_{j}$ at $x, j=1,2,1^{*}, 2^{*}$. Then from (2.11) we have

$$
g\left(\nabla_{X}^{\perp}\left(\sigma\left(E_{a}, E_{a}\right)\right), \sigma\left(E_{a}, E_{a}\right)\right)+g\left(\nabla_{X}^{\perp}\left(\sigma\left(E_{1}, E_{2}\right)\right), \sigma\left(E_{1}, E_{2}\right)\right)=0,
$$
i.e.,

$$
g\left(\nabla_{X}^{\perp}\left(\sigma\left(E_{a}, E_{a}\right)\right), \sigma\left(e_{a}, e_{a}\right)\right)=0 \text { at } x .
$$

Since $\sigma\left(e_{1}, e_{2}\right)=0$ at $x$, we have [5]

$$
\begin{aligned}
\left(\nabla_{X}^{\prime} \sigma\right)\left(e_{a}, e_{a}\right) & =\nabla_{X}^{\perp}\left(\sigma\left(E_{a}, E_{a}\right)\right)-\sigma\left(\nabla_{X} E_{a}, E_{a}\right)-\sigma\left(E_{a}, \nabla_{X} E_{a}\right) \\
& =\nabla_{X}^{\perp}\left(\sigma\left(E_{a}, E_{a}\right)\right)-2 g\left(\nabla_{X} E_{a}, E_{a^{*}}\right) \sigma\left(e_{a}, e_{a^{*}}\right),
\end{aligned}
$$

where $\nabla^{\prime}$ denotes covariant differentiation with respect to the connection in (tangent bundle) $\oplus$ (normal bundle). Hence, we obtain

$$
g\left(\left(\nabla_{X}^{\prime} \sigma\right)\left(e_{a}, e_{a}\right), \sigma\left(e_{a}, e_{a}\right)\right)=0 .
$$

From (2.12) and $\sigma\left(e_{1}, e_{2}\right)=0$ at $x$ we have

$$
g\left(\nabla_{X}^{\perp}\left(\sigma\left(E_{1}, E_{2}\right)\right), \sigma\left(e_{1}, e_{1}\right)\right)+g\left(\nabla_{X}^{\perp}\left(\sigma\left(E_{1}, E_{2}\right)\right), \sigma\left(e_{2}, e_{2}\right)\right)=0 .
$$

Using (2.11) and

$$
\begin{aligned}
\left(\nabla_{X}^{\prime} \sigma\right)\left(e_{1}, e_{2}\right)= & \nabla_{X}^{\perp}\left(\sigma\left(E_{1}, E_{2}\right)\right)-g\left(\nabla_{X} E_{1}, E_{2}\right) \sigma\left(e_{2}, e_{2}\right) \\
& -g\left(\nabla_{X} E_{1}, E_{2^{*}}\right) \sigma\left(e_{2}, e_{2^{*}}\right)-g\left(\nabla_{X} E_{2}, E_{1}\right) \sigma\left(e_{1}, e_{1}\right) \\
& -g\left(\nabla_{X} E_{2}, E_{1^{*}}\right) \sigma\left(e_{1}, e_{1^{*}}\right),
\end{aligned}
$$

we obtain

$$
\begin{aligned}
& g\left(\left(\nabla_{X}^{\prime} \sigma\right)\left(e_{1}, e_{2}\right), \sigma\left(e_{1}, e_{1}\right)\right)+g\left(\nabla_{X} E_{2}, E_{1}\right)\left\|\sigma\left(e_{1}, e_{1}\right)\right\|^{2} \\
& \quad+g\left(\left(\nabla_{X}^{\prime} \sigma\right)\left(e_{1}, e_{2}\right), \sigma\left(e_{2}, e_{2}\right)\right)+g\left(\nabla_{X} E_{1}, E_{2}\right)\left\|\sigma\left(e_{2}, e_{2}\right)\right\|^{2}=0,
\end{aligned}
$$

i.e.,

$$
g\left(\left(\nabla_{X}^{\prime} \sigma\right)\left(e_{1}, e_{2}\right), \sigma\left(e_{1}, e_{1}\right)\right)+g\left(\left(\nabla_{X}^{\prime} \sigma\right)\left(e_{1}, e_{2}\right), \sigma\left(e_{2}, e_{2}\right)\right)=0 .
$$

Since from (2.14) we obtain

$$
g\left(\left(\nabla_{e_{1}^{\prime}}^{\prime} \sigma\right)\left(e_{1}, e_{2}\right), \sigma\left(e_{1}, e_{1}\right)\right)=g\left(\left(\nabla_{e_{2}}^{\prime} \sigma\right)\left(e_{1}, e_{1}\right), \sigma\left(e_{1}, e_{1}\right)\right)=0,
$$

we have

$$
g\left(\left(\nabla_{e_{2}}^{\prime} \boldsymbol{\sigma}\right)\left(e_{1}, e_{1}\right), \sigma\left(e_{2}, e_{2}\right)\right)=g\left(\left(\nabla_{e_{1}}^{\prime} \boldsymbol{\sigma}\right)\left(e_{1}, e_{2}\right), \sigma\left(e_{2}, e_{2}\right)\right)=0 .
$$

Similarly, from (2.13) we have

$$
g\left(\left(\nabla_{e_{2 *}}^{\prime} \sigma\right)\left(e_{1}, e_{1}\right), \sigma\left(e_{2}, e_{2}\right)\right)=0 .
$$

Hence,

$$
g\left(\left(\nabla_{e_{2}^{\prime}}^{\prime} \sigma\right)\left(e_{1}, e_{1}\right), \sigma\left(e_{1}, e_{1^{*}}\right)\right)=g\left(\left(\nabla_{e_{2}^{\prime}}^{\prime} \sigma\right)\left(e_{1}, e_{1}\right), \sigma\left(e_{2}, e_{2^{*}}\right)\right)=0 .
$$


Thus

$$
\left(\nabla_{e_{2}}^{\prime} \sigma\right)\left(e_{1}, e_{1}\right)=0 \text { at } x
$$

Similarly, we obtain

$$
\left(\nabla_{e_{1}}^{\prime} \sigma\right)\left(e_{2}, e_{2}\right)=0 \text { at } x \text {. }
$$

Since $\alpha \beta\left(\alpha^{2}-\beta^{2}\right) \neq 0, \alpha \beta\left(\alpha^{2}-\beta^{2}\right) \neq 0$ in a neighborhood $U$ of $x$. Using the minimal polynomial of $A_{1}$, we can show that $\left\{X \mid A_{1} X=\gamma X\right\}$ is differentiable (e.g. [4]), where $\gamma$ is the eigenvalue of $A_{1}$. Thus we may assume that $A_{1}$ is diagonalized with respect to $\left\{E_{1}, E_{2}, E_{1^{*}}, E_{2^{*}}\right\}$ in $U$. Then we have

$$
g\left(\sigma\left(E_{1}, E_{1}\right), \sigma\left(E_{2}, E_{2}\right)\right)=0 .
$$

Thus

$$
g\left(\nabla_{X}^{\perp}\left(\sigma\left(E_{1}, E_{1}\right)\right), \sigma\left(E_{2}, E_{2}\right)\right)+g\left(\sigma\left(E_{1}, E_{1}\right), \nabla_{X}^{\perp}\left(\sigma\left(E_{2}, E_{2}\right)\right)\right)=0 .
$$

Hence,

$$
\begin{aligned}
& g\left(\left(\nabla_{e_{1}^{\prime}}^{\prime} \sigma\right)\left(e_{1}, e_{1}\right), \sigma\left(e_{2}, e_{2}\right)\right)=0, \\
& g\left(\left(\nabla_{e_{2}^{\prime}}^{\prime} \sigma\right)\left(e_{2}, e_{2}\right), \sigma\left(e_{1}, e_{1}\right)\right)=0 \quad \text { at } x .
\end{aligned}
$$

Therefore we know that $\nabla^{\prime} \sigma=0$ at $x$. On the other hand, from the equation [6]

$$
\frac{1}{2} \Delta\|\sigma\|^{2}=\left\|\nabla^{\prime} \sigma\right\|^{2}-8 \operatorname{trace}\left(\sum A_{\alpha}^{2}\right)^{2}-\sum\left(\operatorname{trace} A_{\lambda} A_{\mu}\right)^{2}+2 \tilde{c}\|\sigma\|^{2},
$$

we obtain $\|\sigma\|^{2}=0$ [5]. This is a contradiction. We know that the case $\alpha \beta \neq 0$ and $\alpha \beta+a_{11} a_{22}+a_{11^{*}} a_{22^{*}}=0$ cannot occur. By the similar argument with the above case we know that both cases $\alpha \beta=0$ and $\alpha^{2}=\beta^{2}$ cannot occur. This completes the proof.

3. Proofs of Theorems A and B. From Proposition 1 we know that $k \leqslant 1$.

Proposition 2. $N_{1}(x)$ has constant dimension on $M^{2}$.

Proof. If the second fundamental form $\sigma(X, Y)=0$ for all $x \in M^{2}$, then $N_{1}(x)$ has constant dimension 0 , and the proofs are complete.

Suppose that $\sigma(X, Y) \neq 0$ at $x_{0} \in M^{2}$. Let $U$ be a neighborhood of $x_{0}$ on which $\sigma(X, Y) \neq 0$. Then $N_{1}(u), u \in U$, has constant dimension 1 on $U$. Since $M^{2}$ is Einstein,

$$
A_{1}=\left(\begin{array}{llll}
\alpha & & & \\
& \alpha & & \\
& & -\alpha & -\alpha
\end{array}\right),
$$

where $\alpha=(1 / 2 \sqrt{2})\|\sigma\|$. This implies $\alpha$ is constant on $U$. Consider the set $S$ defined by

$$
S=\left\{x \in M^{2} \mid \alpha(x)=\alpha\left(x_{0}\right)\right\} .
$$

Since $\alpha$ is continuous on $M^{2}$, we know $S$ is closed. However, the above argument 
implies $S$ is open. Since $x_{0} \in S$, we know $S \neq \varnothing$; so the connectedness of $M^{2}$ implies $S=M^{2}$. Hence, $\alpha=\alpha\left(x_{0}\right)$ on $M^{2}$, and $N_{1}(x)$ has constant dimension 1 on $M^{2}$.

In the case where $N_{1}(x)$ has constant dimension $0, M^{2}$ is totally geodesic. To complete the proofs of Theorems $\mathrm{A}$ and $\mathrm{B}$, we must show that when $N_{1}(x)$ has constant dimension 1 , we can reduce to codimension 1 .

Let $U$ be any coordinate neighborhood of $M^{2}$. We choose orthonormal normal vector fields $\xi_{1}, \xi_{2}$ on $U$ so that $\xi_{1}, \xi_{2}, J \xi_{1}, J \xi_{2}$ span $T_{u}^{\perp}\left(M^{2}\right)$ for any $u \in U$ and such that $\xi_{1}, J \xi_{1}$ span $N_{1}(u)$ for any $u \in U$. We then prove

Proposition 3. For any $x \in U$ and $X \in T_{x}\left(M^{2}\right)$ the following equations are true:

(i) $\nabla_{X}^{1} \xi_{1}=t_{11}(X) J \xi_{1}$,

(ii) $\nabla_{X}^{\perp} \xi_{2}$ and $\nabla_{X}^{\perp} J \xi_{2} \in \operatorname{span}\left\{\xi_{2}, J \xi_{2}\right\}$.

Proof. Equation (1.5) says that

$$
\left(\nabla_{X} A_{2}\right) Y-\sum_{\beta=1}^{2} s_{\beta 2}(X) A_{\beta} Y-\sum_{\beta=1}^{2} t_{\beta 2}(X) J A_{\beta} Y
$$

is symmetric in $X$ and $Y$.

Since $A_{2}=0, \nabla_{X} A_{2}=0$ and (1.5) can be written as

$$
s_{12}(X) A_{1} Y+t_{12}(X) J A_{1} Y=s_{12}(Y) A_{1} X+t_{12}(Y) J A_{1} X .
$$

We can choose $X, Y$ linearly independent vectors so that $A_{1} X=\alpha X$ and $A_{1} Y=\alpha Y$, since $\operatorname{dim} M=2$. Then (3.1) becomes

$$
s_{12}(X) \alpha Y+t_{12}(X) \alpha J Y=s_{12}(Y) \alpha X+t_{12}(Y) \alpha J X .
$$

But $X, Y, J X, J Y$ are linearly independent, so (3.2) implies

$$
s_{12}(X)=t_{12}(X)=0 \text {. }
$$

A similar calculation shows that (3.3) holds for a vector $X$, so that $A_{1} X=-\alpha X$, and, hence, (3.3) holds for all $X \in T_{x}\left(M^{2}\right)$. We recall that

$$
\nabla_{X}^{1} \xi_{\beta}=\sum_{\gamma=1}^{2} s_{\gamma \beta}(X) \xi_{\gamma}+\sum_{\gamma=1}^{2} t_{\gamma \beta}(X) J \xi_{\gamma}, \quad \beta=1,2 .
$$

Then $s_{\beta \gamma}=-s_{\gamma \beta}$ and $t_{\beta \gamma}=t_{\gamma \beta}$ and (3.3) imply that for $\beta=1$, (1.1) becomes

$$
\nabla_{X}^{\perp} \xi_{1}=t_{11}(X) J \xi_{1}
$$

proving (i). For the same reasons, for $\beta=2$, (1.1) becomes

$$
\nabla_{X}^{\perp} \xi_{2}=t_{22}(X) J \xi_{2} .
$$

Then $\nabla_{X}^{\perp} J \xi_{2}=J\left(\nabla_{X}^{\perp} \xi_{2}\right)$ and (3.4) proves (ii).

Proposition 3 implies $N_{1}(x)$ and $N_{0}(x)$ are invariant with respect to $\nabla^{\perp}$. Propositions 2 and 3 and the following lemmas complete the proofs of Theorems A and $\mathbf{B}$.

LeMma 4 (CECIL [1]). Let $f: M^{n} \rightarrow \tilde{M}^{n+p}(\tilde{c})$ be a Kaehler immersion of $M^{n}$ into $\tilde{M}^{n+p}(\tilde{c})$. Suppose $N_{1}(x)$ has constant dimension $k$ and is parallel with respect to the normal connection. Then there is a totally geodesic $(n+k)$-dimensional submanifold $\tilde{M}^{n+k}(\tilde{c})$, such that $f\left(M^{n}\right) \subset \tilde{M}^{n+k}(\tilde{c})$. 
LEMMA 5 (CHERN [2] AND SMYTh [8]). Let $M^{n}$ be an Einstein Kaehler hypersurface of $\tilde{M}^{n+1}(\tilde{c})$. If $n \geqslant 2$, then $M$ is totally geodesic in $\tilde{M}$ or $S=(n / 2) \tilde{c} g$, the latter case arising only when $\tilde{c}>0$. Moreover, the immersion is rigid.

Proof. See, for example, [6, p. 95].

\section{BIBLIOGRAPHY}

1. T. E. Cecil, Geometric applications of critical point theory to submanifolds of complex projective space, Nagoya Math. J. 55 (1974), 5-31.

2. S. S. Chern, On Einstein hypersurface in Kaehler manifold of constant holomorphic sectional curvature,

J. Differential Geom. 1 (1967), 21-31.

3. J.-I. Hano, Einstein complete intersections in complex projective space, Math. Ann. 216 (1975), 197-208.

4. Y. Matsuyama, Minimal submanifolds in $S^{N}$ and $R^{N}$, Math. Z. 175 (1980), 275-282.

5. H. Nakagawa \& R. Takagi, On locally symmetric Kaehler submanifolds in a complex projective space, J. Math. Soc. Japan 28 (1976), 638-667.

6. K. Ogiue, Differential geometry of Kaehler submanifolds, Adv. in Math. 13 (1974), 73-114.

7. B. O'Neill, Isotropic and Kaehler immersions, Canad. J. Math. 17 (1965), 907-915.

8. B. Smyth, Differential geometry of complex hypersurfaces, Ann. of Math. (2) 85 (1967), 246-266.

Department of Mathematics, University of California, Berkeley California 94720

Current address: Department of Mathematics, Chuo University, 1-13-27 Kasuga, Bunkyo-ku, Tokyo 112, Japan 